\title{
Free expansion of two-dimensional condensates with a vortex
}

To cite this article: O Hosten et al 2003 J. Phys. B: At. Mol. Opt. Phys. 362455

View the article online for updates and enhancements.

\section{Related content}

- The ground state and vortices in a two-
$\frac{\text { dimensional Bose gas confined in a }}{\text { harmonic trap }}$
B Tanatar
- Solutions of the Gross-Pitaevskii equation
$\frac{\text { in twodimensions }}{\text { M D Lee and S A Morgan }}$
- Vortices in a trapped dilute Bose-Einstein
$\frac{\text { condensate }}{\text { Alexander L Fetter and Anatoly A }}$
Svidzinsky

\section{Recent citations}

- Dimensional crossover in two-dimensional
Bose-Fermi mixtures
A. L. Subai et al
- Two-dimensional boson-fermion mixtures
A. L. Subai et al
- Bose-Einstein Condensates and the
Numerical Solution of the Gross-Pitaevskii
Equation
S. Succi et al

\section{IOP ebooks}

Bringing you innovative digital publishing with leading voices to create your essential collection of books in STEM research. Start exploring the collection - download the first chapter of every title for free. 


\title{
Free expansion of two-dimensional condensates with a vortex
}

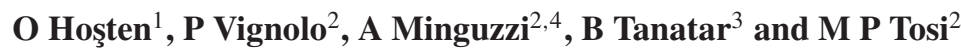 \\ ${ }^{1}$ Department of Physics Engineering, Hacettepe University, Beytepe, 06534 Ankara, Turkey \\ 2 INFM and Classe di Scienze, Scuola Normale Superiore, I-56126 Pisa, Italy \\ ${ }^{3}$ Department of Physics, Bilkent University, Bilkent, 06533 Ankara, Turkey \\ E-mail: minguzzi@sns.it
}

Received 20 February 2003, in final form 31 March 2003

Published 4 June 2003

Online at stacks.iop.org/JPhysB/36/2455

\begin{abstract}
We study the free expansion of a pancake-shaped Bose-condensed gas, which is initially trapped under harmonic confinement and containing a vortex at its centre. In the case of a radial expansion holding the axial confinement fixed we consider various models for the interactions, depending on the thickness of the condensate relative to the value of the scattering length. We are thus able to evaluate different scattering regimes ranging from quasi-three-dimensional (Q3D) to strictly two-dimensional (2D). We find that as the system goes from $\mathrm{Q} 3 \mathrm{D}$ to $2 \mathrm{D}$ the expansion rate of the condensate increases whereas that of the vortex core decreases. In the Q3D scattering regime we also examine a fully free expansion in 3D and find oscillatory behaviour for the vortex core radius: an initial fast expansion of the vortex core is followed by a slowing down. Such a nonuniform expansion rate of the vortex core implies that the timing of its observation should be chosen appropriately.
\end{abstract}

\section{Introduction}

Two-dimensional (2D) condensates in harmonic confinement are attracting a lot of attention. By varying the anisotropy parameter, measured as the ratio between the trap frequencies in the $z$ - and the planar directions, flatter and flatter (pancake-shaped) condensates are being produced in magnetic or optical traps [1-3] with the ultimate goal of observing the special features of low dimensionality. As the gas approaches the $2 \mathrm{D}$ limit, its collisional properties start to influence the boson-boson coupling parameter, which becomes dependent on the density of the system even at low density and zero temperature. This is in contrast to the situation in $3 \mathrm{D}$, where to lowest order in the density the interactions are described by a constant coupling strength and

4 Author to whom any correspondence should be addressed. 
deviations arise only when quantum depletion and finite-temperature effects have to be taken into account [4].

The quantized vortex states are important in establishing the superfluid nature of the condensates [5-9]. Many current experiments on vortices [10,11] have utilized elongated geometries, but some observations on flat condensates have already been reported $[12,13]$ and one may expect that further studies on pancake-shaped condensates will follow. Understanding vortices in $2 \mathrm{D}$ is important since they are expected to play a role in the transition from the superfluid to the normal state [14]. Their role in inhomogeneous systems such as trapped gases is less well known.

From the experimental point of view, observing a vortex in a trapped gas is difficult because of the smallness of the core region compared to the size of the gaseous cloud. Several solutions have been suggested to circumvent this problem. The size of the core relative to the cloud radius is larger in systems made of a smaller number of particles. Another possibility is to create vortices with large angular momentum as achieved in a recent experiment [15]. The vortex core increases in a free expansion after the release of the trap. Indeed, it has been suggested [7] that the vortex core may expand faster than the condensate cloud. A common method for the observation of vortices is based on releasing the trap and allowing the condensate to expand [10].

Several theoretical works have studied the free expansion of vortices in Bose-condensed systems inside anisotropic trap potentials employing various 3D models for the coupling constant $[7,8,16]$. In a recent work [17] we have calculated the equilibrium density profiles of pancake-shaped condensates using various models for the coupling and we have found that, when the condensate is in the $2 \mathrm{D}$ regime as regards its collisional properties, its density profile is very different in size from what is predicted by the 3D model.

We treat in this paper pancake-shaped condensates with a vortex on the basis of various models for the coupling parameters, depending on the thickness of the condensate relative to the value of the scattering length. As this parameter is decreased the scattering evolves from quasi-three-dimensional (Q3D) to quasi-two-dimensional (Q2D) and to strictly twodimensional (2D). We study both the equilibrium profile and the expansion properties of the condensate after the trapping potential is released. By calculating the time evolution of the condensate size and of the vortex core radius and their expansion rates in $2 \mathrm{D}$ we again establish how the crossover regime may be identified in such experiments. We find that the condensate cloud expands faster in its plane as the system goes from being Q3D to 2D. The expansion rate of the vortex core radius, on the other hand, decreases in the dimensionality crossover. We also find that the expansion characteristics of the condensate cloud and of the vortex core are rather different when the trap potential is released only in the radial plane or in both the $z$ - and the perpendicular directions. In the latter case the velocity of the vortex core can be greater than that of the condensate cloud in the initial phase of the expansion.

The paper is organized as follows. We first introduce our description of condensates with a vortex for different regimes of scattering properties in section 2 . We then present our results in section 3 and conclude with a brief summary in section 4 .

\section{The model}

We consider a dilute Bose-condensed gas at zero temperature under anisotropic harmonic confinement characterized by a trap frequency $\omega_{\perp}$ in the $x-y$ plane and an axial trap frequency $\omega_{z}=\lambda \omega_{\perp}$, with $\lambda \gg 1$. The motion in the $z$-direction is frozen and we may describe the ground state of the condensate by the wavefunction $\psi(r)$ in the $x-y$ plane, to be determined from a 2D Schrödinger equation. The dynamics of the 2D condensate which accommodates 
a quantized vortex state is described by a time-dependent nonlinear Schrödinger equation (NLSE), which reads

$\mathrm{i} \hbar \frac{\partial}{\partial t} \psi(r, t)=\left[-\frac{\hbar^{2}}{2 m} \nabla^{2}+\frac{\hbar^{2} \kappa^{2}}{2 m r^{2}}+\frac{1}{2} m \omega_{\perp}^{2} r^{2}+g(\psi(r, t))|\psi(r, t)|^{2}\right] \psi(r, t)$.

Here, $\hbar \kappa$ is the quantized angular momentum along the $z$-axis, $m$ is the atomic mass, and $g(\psi)$ is the coupling strength in $2 \mathrm{D}$ which depends on the condensate wavefunction as discussed below. We have assumed that the order parameter entering the NLSE can be written as $\Phi(\boldsymbol{r}, t)=\psi(r, t) \mathrm{e}^{\mathrm{i} \kappa \phi}$ where $\phi$ is the azimuthal angle. We consider repulsive interactions between the atoms in the condensate, which enter the coupling strength $g$ through a positive scattering length $a$.

As we have discussed previously [17], the density profile of the condensate reflects the modified shape of the confining potential and the modified scattering properties. When the linear dimension $a_{z}=\left(\hbar / m \omega_{z}\right)^{1 / 2}$ of the condensate cloud in the $z$-direction is much larger than the 3D scattering length $\left(a_{z} \gg a\right)$, the collisions take place in three dimensions and the coupling constant to be used in the NLSE is

$$
g_{\mathrm{Q} 3 \mathrm{D}}=2 \sqrt{2 \pi} \frac{\hbar^{2}}{m} \frac{a}{a_{z}}
$$

including only the geometrical effects of the reduced dimensionality.

When the anisotropy further increases and $a_{z}$ becomes comparable with $a\left(a_{z} \simeq a\right)$, the collisions start to be influenced by the confinement in the $z$-direction and the interaction strength assumes a form appropriate to a Q2D condensate:

$$
g_{\mathrm{Q} 2 \mathrm{D}}=\frac{2 \sqrt{2 \pi}\left(\hbar^{2} / m\right)\left(a / a_{z}\right)}{1+\frac{a}{\sqrt{2 \pi} a_{z}}\left|\ln \left(g_{\mathrm{Q} 2 \mathrm{D}} n\left(2 \pi m / \hbar^{2}\right) a_{z}^{2}\right)\right|} .
$$

Here, $n(r, t)=N|\psi(r, t)|^{2}$ is the areal density of the condensate cloud, whose dependence on the radial coordinate and on time is to be calculated self-consistently during the numerical solution of equation (1). This expression was originally derived by Petrov et al [18, 19] by studying the scattering amplitude in a system which is harmonically confined in the $z$ direction and homogeneous in the $x-y$ plane. The same result has also been obtained by Lee et al $[20,21]$ within a many-body $T$-matrix approach. Note that the coupling strength now depends on density, as is to be expected for $2 \mathrm{D}$ collisions. Furthermore, $g_{\mathrm{Q} 2 \mathrm{D}}$ is given by an implicit relation which has to be solved numerically during the solution of the NLSE ${ }^{5}$.

Finally, the strictly 2D regime is approached as $a_{z}$ becomes much smaller than $a\left(a_{z} \ll a\right)$ and the system is described by the coupling

$$
g_{2 \mathrm{D}}=\frac{4 \pi \hbar^{2}}{m} \frac{1}{\left|\ln \left(n a^{2}\right)\right|},
$$

as was first derived by Schick [22] for a homogeneous Bose gas of hard discs ${ }^{6}$. The use of the coupling strength $g_{2 \mathrm{D}}$ for inhomogeneous systems, involving a dependence on the local density,

5 In our previous work [17] we have approximated the expression given in equation (3) by $g_{\mathrm{Q} 2 \mathrm{D}}=$ $2 \sqrt{2 \pi}\left(\hbar^{2} / m\right)\left(a / a_{z}\right) /\left[1+\left(a / \sqrt{2 \pi} a_{z}\right)\left|\ln \left(2(2 \pi)^{3 / 2} n a a_{z}\right)\right|\right]$, which amounts to approximating $g_{\mathrm{Q} 2 \mathrm{D}}$ inside the logarithm in equation (3) by its zeroth-order value $2 \sqrt{2 \pi}\left(\hbar^{2} / m\right) a / a_{z}$. We have checked that by using equation (3) the main results of [17] are not altered to any significant extent. Indeed, the implicit expression for $g_{\mathrm{Q} 2 \mathrm{D}}$ can change the wavefunction profiles only for extremely large values of the anisotropy parameter $\lambda$.

6 In the definition of $g_{2 \mathrm{D}}$ we are using the 3D scattering length $a$. The work of Petrov and Shlyapnikov [19] and that of Lee and Morgan [21] indicate that the use of the 2D scattering length $a_{2 D}$ would be more appropriate, but since for a $2 \mathrm{D}$ system $a_{2 \mathrm{D}}=a \mathrm{e}^{\gamma}$ (where $\gamma \simeq 0.577$ is the Euler-Mascheroni constant), in the dilute-gas regime $\left(n a^{2} \ll 1\right)$ the difference between the two models goes beyond the accuracy of the mean-field description. 
has been proposed by Shevchenko [23] and more recently by Kolomeisky et al [24]. Note that also $g_{2 \mathrm{D}}$ has a spatial dependence due to $n$ but, unlike $g_{\mathrm{Q} 2 \mathrm{D}}$, carries no information on the confinement in the $z$-direction.

We solve the time-dependent NLSE iteratively by discretization using a split-step CrankNicholson scheme [25]. In the case of 2D calculations a simple one-dimensional array of grid points is sufficient to describe the spatial part of $\psi(r, t)$ at each time step. The 3D calculations that we subsequently report for $\psi(r, z, t)$ as a solution of the standard Gross-Pitaevski equation (GPE) involve a 2D grid because of the cylindrical symmetry of this wavefunction.

\section{Results and discussion}

\subsection{Equilibrium profiles and radial expansion}

The numerical solution of the NLSE with a centrifugal term in equilibrium conditions gives the ground-state wavefunction of the condensate with a single vortex $(\kappa=1)$. We first take values of the anisotropy parameter and of the scattering length as appropriate for ${ }^{23} \mathrm{Na}$ atoms in the experiment of Görlitz et al $[1]\left(\lambda=26.33\right.$ and $a=2.8 \mathrm{~nm}$, so $\left.a / a_{z}=3.8 \times 10^{-3}\right)$. We scale the radial coordinate by the harmonic oscillator length $a_{\perp}=\left(\hbar / m \omega_{\perp}\right)^{1 / 2}$ and the radial wavefunction by $1 / a_{\perp}$. We take $N=5000$ since it is easier to observe vortices for smaller numbers of particles. For these parameters, the system undergoes collisions in $3 \mathrm{D}$ but has $2 \mathrm{D}$ characteristics as regards the confinement effects. As in the absence of the vortex, we find that the Q3D and Q2D models yield wavefunction profiles identical to each other as shown in figure 1(a), whereas the 2D model produces a quantitatively very different result. Evidently, the 2D model is physically incorrect in this regime of parameters.

We next look at the planar free expansion of the condensate with a vortex for the same parameters, holding fixed the confinement in the axial direction. The time-dependent NLSE is solved after the trap potential $\frac{1}{2} m \omega_{\perp}^{2} r^{2}$ is switched off at time $t=0$. Figure 1 (b) shows the time dependence of the root mean square (rms) value of the radial coordinate $r_{\mathrm{rms}}=\left\langle r^{2}\right\rangle^{1 / 2}$, which describes the size of the condensate cloud, and of the vortex core radius $R_{\mathrm{c}}$ in three different models of the coupling. As in [8], the core radius is chosen to be the value of $r$ where the condensate density reaches $1 / \mathrm{e}$ of its peak value, namely $\left|\psi\left(R_{\mathrm{c}}\right)\right|^{2}=\mathrm{e}^{-1} \max \left\{|\psi(r)|^{2}\right\}$. We see from figure 1(b) that the Q3D and Q2D models again yield identical results for the present set of parameters. The vortex core expands much more slowly than the condensate cloud, as indicated in the same figure. We also show in figure 1(c) the velocity of expansion of the atomic cloud and of the vortex core as functions of time. After an initial accelerated motion both velocities attain a constant value for $t \omega_{\perp} \gtrsim 2$.

We increase the anisotropy parameter to $\lambda=2 \times 10^{5}$ and take $a / a_{z}=0.33$ in order to investigate the regime of crossover from $3 \mathrm{D}$ to $2 \mathrm{D}$. As shown in figure $2(\mathrm{a})$, the profiles of the wavefunction with a vortex all look similar in this regime of parameters. Consequently the time dependence of the radial coordinate and of the vortex core radius display similar behaviours during the expansion, as shown in figure 2(b). We note that the expansion of the condensate cloud is faster than in the Q3D regime. This is also indicated by the higher velocities depicted in figure 2(c). The velocity of the vortex core radius, however, decreases compared to the previous regime.

The system enters the strictly 2D regime when we further increase the scattering length to $a / a_{z}=2.68$. In this case, the Q3D model becomes physically incorrect and the vortex wavefunction is best described by the $2 \mathrm{D}$ model using $g_{2 \mathrm{D}}$, although the results from the Q2D model using $g_{\mathrm{Q} 2 \mathrm{D}}$ are very similar. As the initial shape of the cloud in the Q3D model extends out to a larger radial distance as shown in figure 3(a), one may again distinctly identify the 

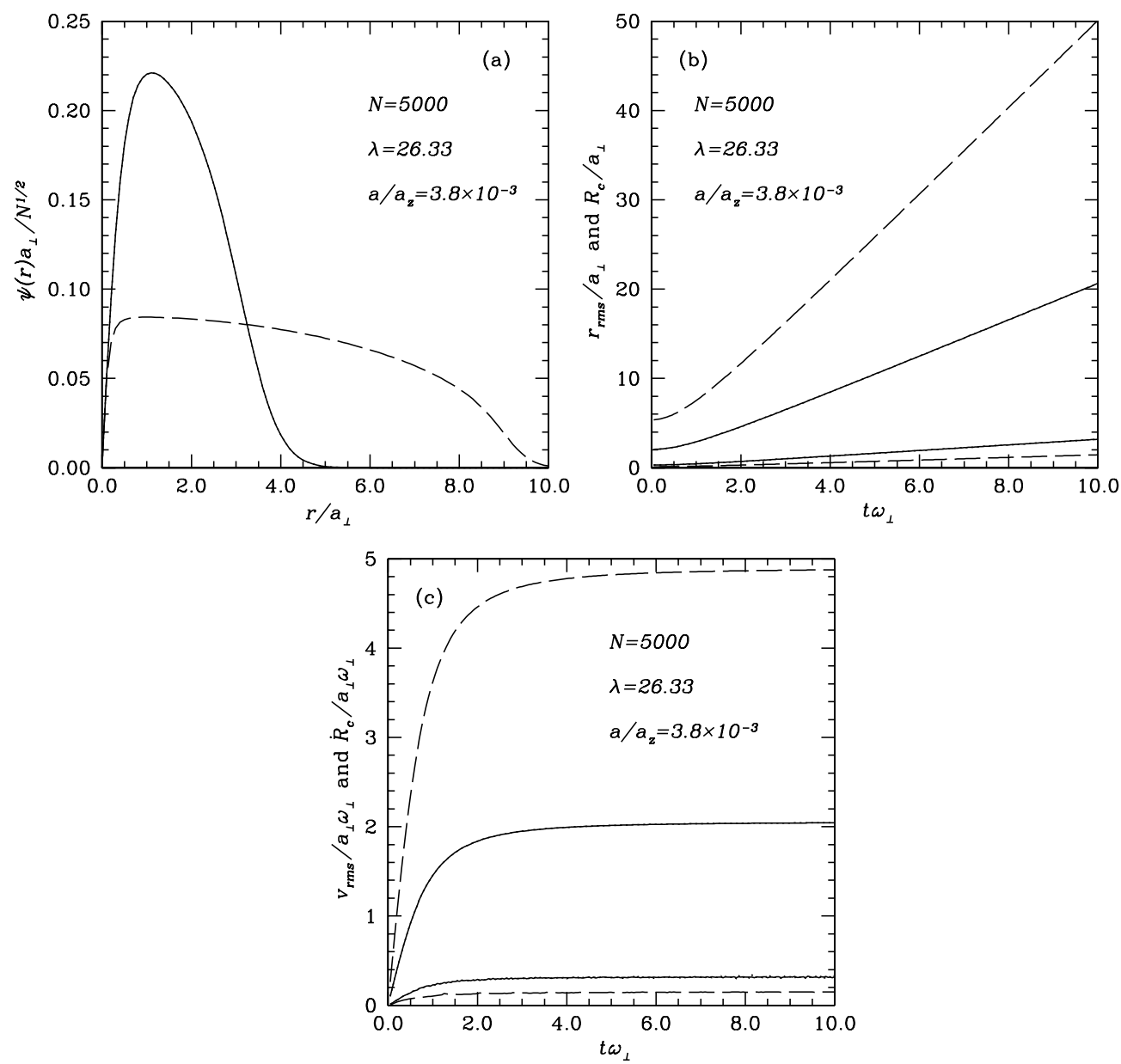

Figure 1. Properties of a condensate with a vortex within the models described by $g_{\mathrm{Q} 3 \mathrm{D}}$ (dotted curves), $g_{\mathrm{Q} 2 \mathrm{D}}$ (solid curves), and $g_{2 \mathrm{D}}$ (dashed curves), for $N=5000, \lambda=26.33$, and $a / a_{z}=3.8 \times 10^{-3}$. The dotted curves are superimposed on the solid curves for this set of parameters. (a) The vortex-state equilibrium wavefunction. (b) The time dependence of the rms value $r_{\text {rms }}$ of the radial coordinate (upper curves) and that of the vortex core radius $R_{\mathrm{c}}$ (lower curves). (c) The time dependence of the rms value $v_{\text {rms }}$ of the velocity of the radial coordinate (upper curves) and that of the velocity $\dot{R}_{\mathrm{c}}$ of the vortex core radius (lower curves).

truly $2 \mathrm{D}$ regime by the wavefunction profile. Furthermore, we observe in figure $3(\mathrm{~b})$ that the atomic cloud expands slightly faster as compared to the previous Q3D and crossover regimes. This is also evident in figure 3(c), where the expansion velocity of the cloud is larger. The velocity $\dot{R}_{\mathrm{c}}$ of the vortex core radius, on the other hand, is very small in the strictly $2 \mathrm{D}$ regime.

The above results indicate that the initially trapped Bose condensate expands faster in the $x-y$ plane as the system moves from being 3D to 2D. The opposite behaviour is observed for the vortex core. However, the expansion rate of the condensate cloud remains larger than that of the vortex in all cases that we have considered. These behaviours are evidently a consequence of the increasing repulsions, as is also apparent from the reduced healing length of the condensate before expansion is allowed. Our numerical results for the Q3D regime are consistent with previous studies of vortices in Bose-condensed fluids [7, 8] for similar values of the parameters. 

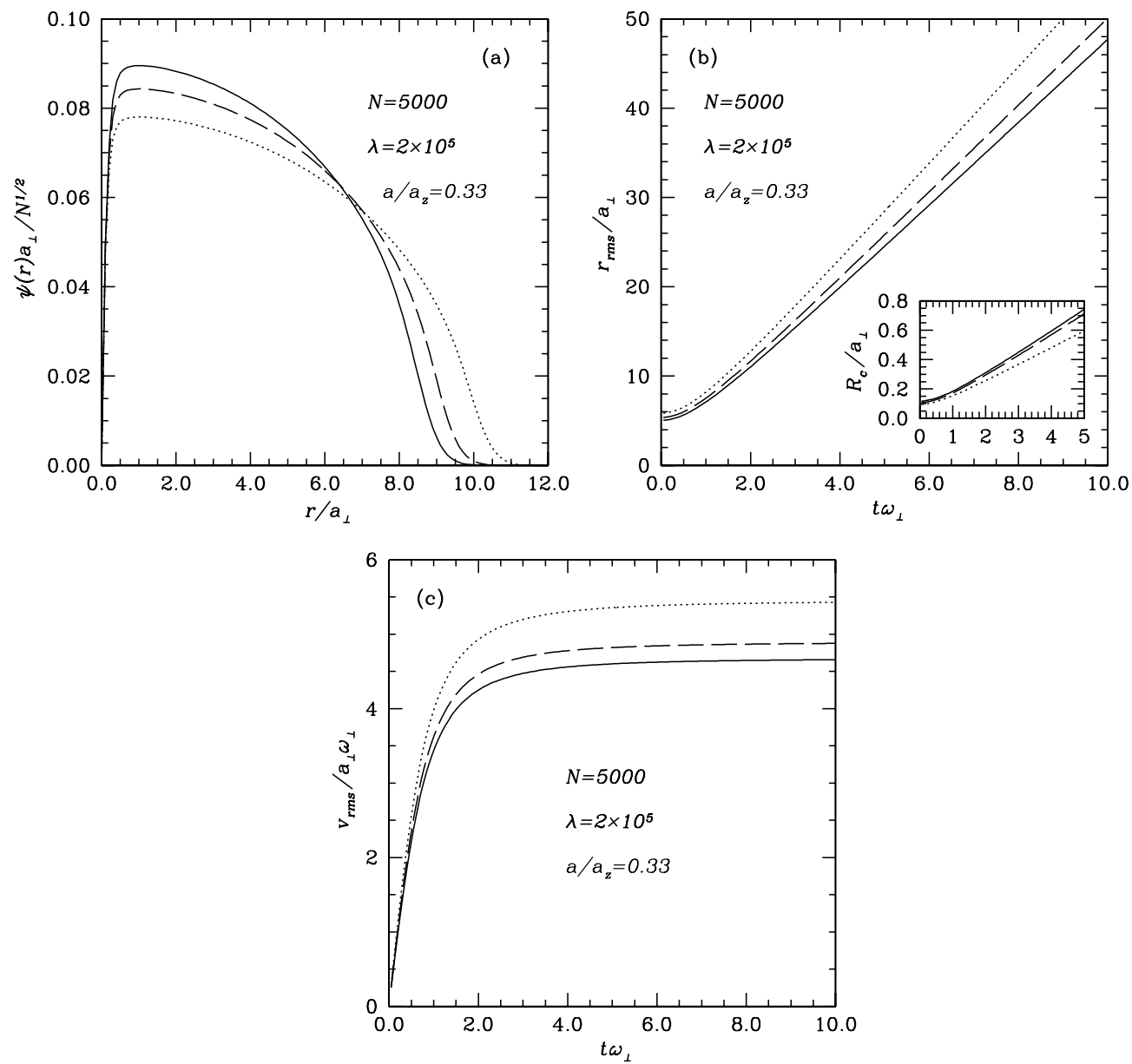

Figure 2. Properties of a condensate with a vortex within the models described by $g_{\mathrm{Q} 3 \mathrm{D}}$ (dotted curves), $g_{\mathrm{Q} 2 \mathrm{D}}$ (solid curves), and $g_{2 \mathrm{D}}$ (dashed curves), for $N=5000, \lambda=2 \times 10^{5}$, and $a / a_{z}=0.33$. (a) The vortex-state wavefunction at equilibrium. (b) The time dependence of rms radial coordinate $r_{\text {rms }}$ and of the vortex core radius $R_{\mathrm{c}}$ (inset). (c) The time dependence of the rms velocity $v_{\text {rms }}$ of the radial coordinate.

\subsection{Three-dimensional expansion}

We focus now on the case of a free expansion in 3D space, adopting the set of experimentally relevant parameters as in figure 1. In this case the presence of the confinement does not affect the binary collisions between the atoms and the system is well described by the 3D time-dependent GPE with coupling constant $g_{3 \mathrm{D}}=4 \pi \hbar^{2} a / m$.

We have first tested the consistency of our Q3D model by solving the GPE for a 3D anisotropic system to study the expansion when only the radial trap potential $m \omega_{\perp}^{2} r^{2} / 2$ is turned off (i.e. the potential $m \omega_{z}^{2} z^{2} / 2$ remains throughout the expansion). The results are identical to those obtained earlier in figure 1 with the 2D kinematics and the Q3D scattering properties, showing the correctness of our physical picture.

We then studied the expansion properties of a 3D anisotropic condensate when the trap potentials in both $r$ - and $z$-directions are released. Essentially identical results are obtained for 

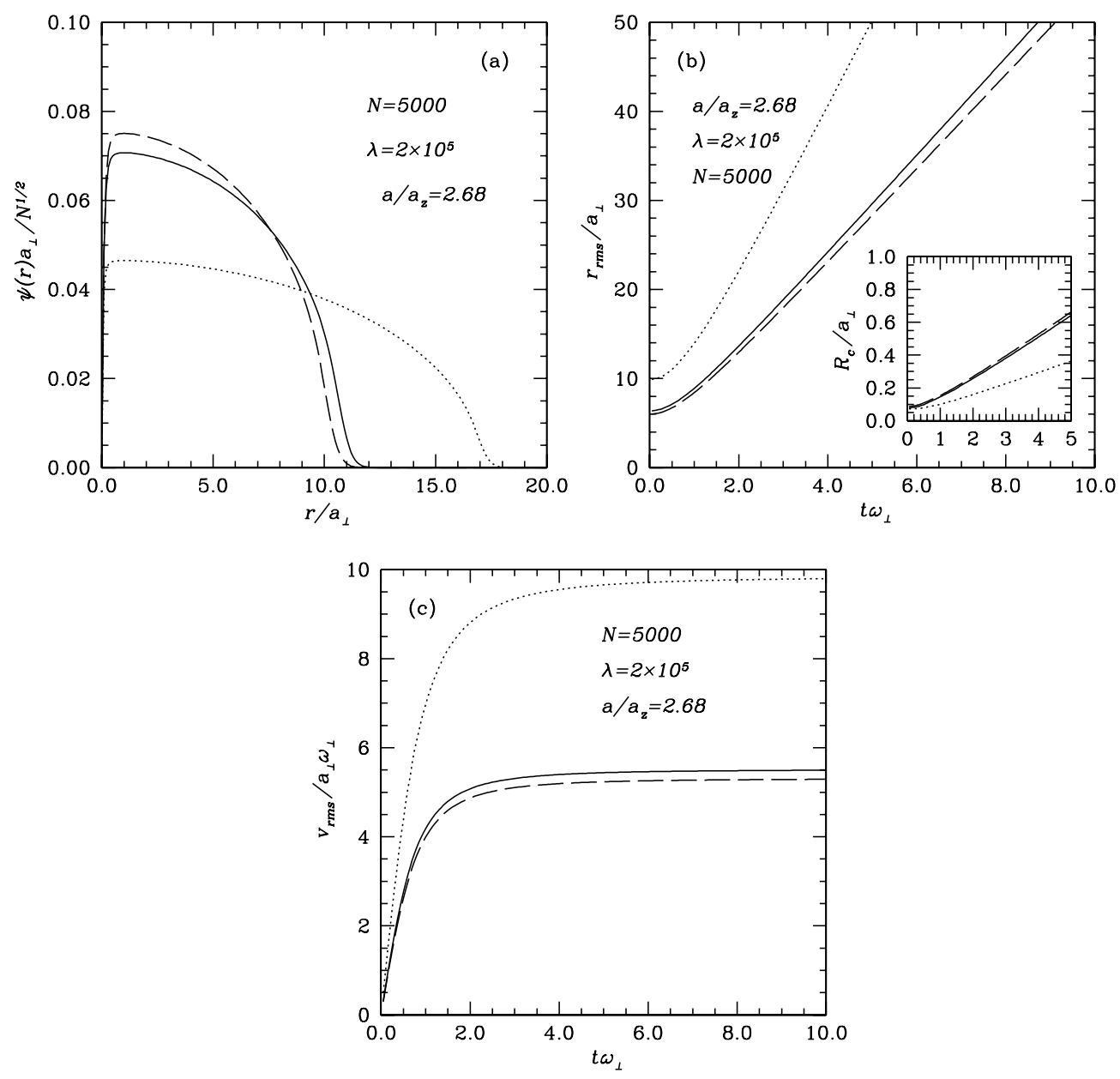

Figure 3. Properties of a condensate with a vortex within the models described by $g_{\mathrm{Q} 3 \mathrm{D}}$ (dotted curves), $g_{\mathrm{Q} 2 \mathrm{D}}$ (solid curves), and $g_{2 \mathrm{D}}$ (dashed curves), for $N=5000, \lambda=2 \times 10^{5}$, and $a / a_{z}=2.68$. (a) The vortex-state wavefunction at equilibrium. (b) The time dependence of the rms radial coordinate $r_{\text {rms }}$ and of the vortex core radius $R_{\mathrm{c}}$ (inset). (c) The time dependence of the rms velocity $v_{\text {rms }}$ of the radial coordinate.

two different choices of the initial configuration, i.e. starting from the ground state of the 3D GPE or from the ground state of the 2D GPE with Q3D coupling combined with a Gaussian profile in the third direction. In both cases we calculate the time-dependent behaviour of the rms values of the radial and axial coordinates, as well as that of the core radius $R_{\mathrm{c}}$. The results are shown in figure 4 . We observe a much faster expansion for the $z$-coordinate, reflecting the initially tighter axial confinement. It also appears that the vortex core radius undergoes a slow oscillation, as is more evident in figure 4(b) where we plot the velocities.

The oscillatory behaviour of the vortex core can be understood as being closely related to the repulsive interactions, which strongly affect the density profile. As compared to the ground state of the noninteracting case, the repulsive interactions give rise to a smaller vortex core radius and to a larger rms radius. The density suddenly decreases when the trap is released in both $r$-and $z$-directions, as a result of the fast expansion in the $z$-direction, and this causes the nonlinear interaction energy to vanish on the timescale of the radial expansion. Therefore, in 

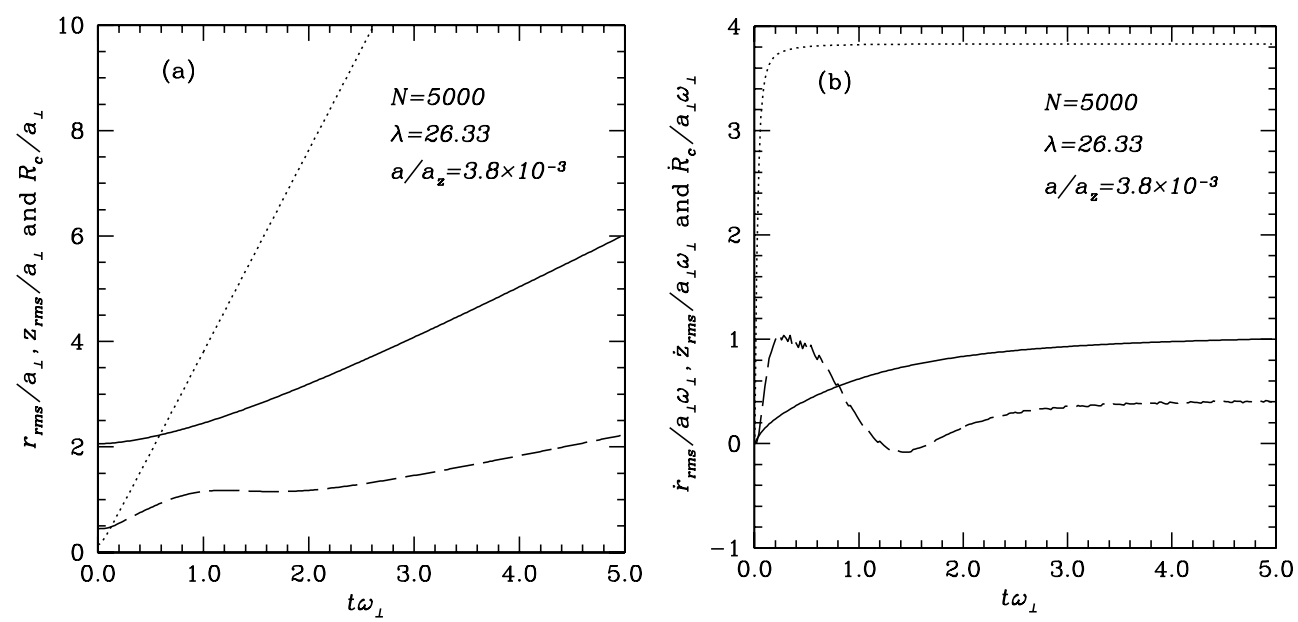

Figure 4. (a) The time dependence of the rms values of the radial (solid curves) and $z$-coordinates (dotted curves), and that of the vortex core radius (dashed curves), for a 3D system with $N=5000$, $\lambda=26.33$, and $a / a_{z}=3.8 \times 10^{-3}$. (b) The time dependence of the velocities $\dot{r}_{\mathrm{rms}}, \dot{z}_{\mathrm{rms}}$, and $\dot{R}_{\mathrm{c}}$ for the same system.

the first part of the expansion (for $t \omega_{\perp} \lesssim 0.8$ in our study) the compressed density at the centre of the cloud expands faster than the remaining part, with the result that the core expansion rate is larger than that of the condensate cloud. Similar results were also found by Dalfovo and Modugno [8]. At larger times the expansion of the vortex slows down while the radial expansion of the cloud becomes ballistic. The velocity $\dot{R}_{\mathrm{c}}$ of the vortex core even reverses sign around $t \omega_{\perp} \approx 1.5$, as shown in figure $4(\mathrm{~b})$.

We have also studied the expansion properties of systems with $N=5 \times 10^{4}$ and $5 \times 10^{5}$ particles and the same trap frequencies and scattering length as in figure 1, finding again a very rapid expansion in the $z$-direction and oscillatory behaviour in the vortex core radius. These results are not shown since they are qualitatively very similar to those in figure 4 .

\section{Summary and concluding remarks}

From the perspective of experimental investigations on low-dimensional condensates, we have studied the free expansion of pancake-shaped Bose-Einstein condensates with a vortex. By choosing various values of the trap anisotropy and scattering length we have explored different scattering regimes, from a $\mathrm{Q} 3 \mathrm{D}$ regime to a strictly $2 \mathrm{D}$ one. We have described the cloud at the mean-field level, taking as coupling strengths for the boson-boson interactions the densitydependent expressions derived by Petrov et al [18] for the quasi-2D regime and by Kolomeisky et al [24] for the strictly 2D regime.

We have considered both the case of a 2D expansion keeping the confinement along $z$ fixed and the case of a fully $3 \mathrm{D}$ expansion. In the $2 \mathrm{D}$ case we have observed that with increasing anisotropy the expansion rate of the cloud increases while that of the vortex core decreases. In the 3D expansion, performed in the regime where the collisions are three-dimensional and the Q3D model is the most appropriate, the expansion rate of the vortex core exhibits an oscillatory behaviour due to the interplay between nonlinear interactions and anisotropic confinement. Such a nonuniform expansion rate of the vortex core implies that the timing of its observation is an important parameter. 
Whereas in this work we have restricted our analysis to the case of a condensate with a single vortex at $T=0$, it may also be interesting to investigate the role of the noncondensate cloud at finite temperature, as vortices are intimately connected with the Kosterlitz-Thouless phase transition. To explore this area it would be necessary to take into account multiple vortices within the condensate cloud [9].

\section{Acknowledgments}

We acknowledge partial support by INFM within the PRA-Photonmatter Initiative. OH thanks TUBITAK-BAYG. BT acknowledges the support of TUBITAK, NATO-SfP, MSB-KOBRA, and TUBA, and the hospitality of Scuola Normale Superiore during part of this work.

\section{References}

[1] Görlitz A et al 2001 Phys. Rev. Lett. 87130402

[2] Zobay O and Garraway B M 2001 Phys. Rev. Lett. 861195

[3] Gauck H, Hartl M, Schneble D, Schnitzler H, Pfau T and Mlynek J 1998 Phys. Rev. Lett. 815298 Hinds E A, Boshier M G and Hughes I G 1998 Phys. Rev. Lett. 80645

[4] Hutchinson D A W, Dodd R J and Burnett K 1998 Phys. Rev. Lett. 812198

[5] Leggett A J 1999 Rev. Mod. Phys. 71 S318

[6] Dalfovo F and Stringari S 1996 Phys. Rev. A 532477

[7] Lundh E, Pethick C J and Smith H 1998 Phys. Rev. A 584816

[8] Dalfovo F and Modugno M 2000 Phys. Rev. A 61023605

[9] Castin Y and Dum R 1999 Eur. Phys. J. D 7399

[10] Madison K W, Chevy F, Wohlleben W and Dalibard J 2000 Phys. Rev. Lett. 84806

[11] Anderson B P, Haljan P C, Wieman C E and Cornell E A 2000 Phys. Rev. Lett. 852857 Abo-Shaeer J R, Raman C, Vogels J M and Ketterle W 2001 Science 292476

[12] Hodby E, Hechenblaikner G, Hopkins S A, Maragó and Foot C J 2002 Phys. Rev. Lett. 88010405

[13] Haljan P C, Coddington I, Engels P and Cornell E A 2001 Phys. Rev. Lett. 87210403

[14] Kosterlitz J M and Thouless D J 1973 J. Phys. C: Solid State Phys. 61181

[15] Leanhardt A E, Görlitz A, Chikkatur A P, Kielpinski D, Shin Y, Pritchard D E and Ketterle W 2002 Phys. Rev. Lett. 89190403

[16] Adhikari S K 2002 Phys. Rev. A 65033616 Adhikari S K 2002 Phys. Rev. A 66043601

[17] Tanatar B, Minguzzi A, Vignolo P and Tosi M P 2002 Phys. Lett. A 302131

[18] Petrov D S, Holzmann M and Shlyapnikov G V 2000 Phys. Rev. Lett. 842551

[19] Petrov D S and Shlyapnikov G V 2001 Phys. Rev. A 64012706

[20] Lee M D, Morgan S A, Davis M J and Burnett K 2002 Phys. Rev. A 65043617

[21] Lee M D and Morgan S A 2002 J. Phys. B: At. Mol. Opt. Phys. 353009

[22] Schick M 1971 Phys. Rev. A 31067

[23] Shevchenko S I 1991 Sov. Phys.-JETP 731009 Shevchenko S I 1992 Sov. J. Low Temp. Phys. 18223

[24] Kolomeisky E B, Newman T J, Straley J P and Qi X 2000 Phys. Rev. Lett. 851146

[25] Press W H, Teukolsky S A, Vettering W T and Flannery P B 1992 Numerical Recipes 2nd edn (Cambridge: Cambridge University Press) 